\title{
Statins Stimulate Hepatic Glucose Production via the miR-183/96/182 Cluster
}

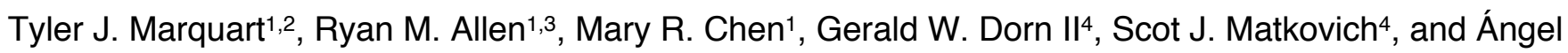
Baldán ${ }^{1,5,6}$

'Edward A. Doisy Department of Biochemistry and Molecular Biology, Saint Louis University, 1100 S.

Grand Blvd., Saint Louis, MO 63104

${ }^{2}$ Current address: PierianDx Inc., 77 Maryland Plaza, Saint Louis, MO 63108

${ }^{3}$ Current address: Department of Medicine, Vanderbilt University Medical Center, 2220 Pierce Ave.,

312B PRB, Nashville, TN 37232

${ }^{4}$ Center for Pharmacogenomics, Department of Internal Medicine, Washington University School of

Medicine, Saint Louis, MO 63110

${ }^{5}$ Center for Cardiovascular Research, Saint Louis University

${ }^{6}$ Liver Center, Saint Louis University

Keywords: SREBP, miRNA, TCF7L2, gluconeogenesis, diabetes 


\section{Contact Information}

Ángel Baldán

Saint Louis University School of Medicine

Doisy Research Center, room 615

1100 S. Grand Blvd.

Saint Louis, MO 63104

phone: +1.314 .977 .9227$

e-mail: angel.baldan@health.slu.edu 
Statins are the most common pharmacologic intervention in hypercholesterolemic patients, and their use is recognized as a key medical advance leading to a $\mathbf{5 0 \%}$ decrease in deaths from heart attack or stroke over the past $\mathbf{3 0}$ years. The atheroprotective outcomes of statins are largely attributable to the accelerated hepatic clearance of low-density lipoprotein (LDL)-cholesterol from circulation, following the induction of the LDL receptor. However, multiple studies suggest that these drugs exert additional LDL-independent effects. The molecular mechanisms behind these so-called pleiotropic effects of statins, either beneficial or undesired, remain largely unknown. Here we determined the coding transcriptome, miRNome, and RISCome of livers from mice dosed with saline or atorvastatin to define a novel in vivo epitranscriptional regulatory pathway that links statins to hepatic gluconeogenesis, via the SREBP2-miR-183/96/182-TCF7L2 axis. Notably, multiple genome-wide association studies identified TCF7L2 (transcription factor 7 like 2) as a candidate gene for type 2 diabetes, independent of ethnicity. Conclusion: our data reveal an unexpected link between cholesterol and glucose metabolism, provides a mechanistic explanation to the elevated risk of diabetes recently observed in patients taking statins, and identifies the miR-183/96/182 cluster as an attractive pharmacological candidate to modulate noncanonical effects of statins.

Statins are competitive inhibitors of 3-hydroxy-3-methyl-glutaryl-coenzyme A reductase (HMGCR), the rate-limiting enzyme in the cholesterol biosynthetic pathway (1). The ensuing decrease in intracellular sterol levels triggers the proteolytic maturation of the sterol regulatory element-binding protein 2 (SREBP2), which orchestrates a transcriptional program that increases both de novo synthesis and uptake of cholesterol. Among the targets of SREBP2, the LDLR is critical in mediating the reduction in plasma LDL-cholesterol which reduces cardiovascular risk in patients taking statins (2). Meta-analyses of epidemiological data and studies in animal models and cells have demonstrated LDL-cholesterol independent effects of statins, both beneficial (improved endothelial function, decreased vascular 
inflammation, reduced thrombogenic response) and undesired (myalgia, liver damage, memory loss) (3). Importantly, statin therapy is modestly, but significantly, associated dose-dependently with increased new-onset diabetes (reviewed in $(4,5)$ ). Indeed, in 2012 the Federal Drug Administration mandated a modification of the safety label of statins to warn of increased $\mathrm{HbA1c}$ and/or fasting plasma glucose. This correlation might be the result of prescription bias, i.e. patients who are at increased risk of developing diabetes are also more prone to dyslipidemias and, consequently, are prescribed statins more frequently; however, different laboratories demonstrated decreased pancreatic $\beta$-cell insulin secretion (6), peripheral insulin sensitivity $(7,8)$, and skeletal muscle mitochondrial function $(9,10)$ following treatment with statins in vitro and/or in vivo, thus suggesting that statins per se do indeed impact glucose homeostasis. The role of statins on hepatic glucose production, however, has never been examined.

MicroRNAs (miRs) are small, noncoding 20-22 nt RNAs that bind to specific, partially complementary sequences in the $3^{\prime}$ untranslated region (UTR) of target mRNAs and recruit them to the RNA-induced silencing complex (RISC) to promote their cleavage and/or translational repression (11). Over the last two decades, miRNAs have been recognized as key regulators of multiple (patho)physiological processes as their expression can be tissue-, developmental-, and disease-specific (12). In the context of cholesterol metabolism, we and others reported on miR-33a (reviewed in (13)): an intragenic miRNA encoded within intron 16 of SREBP2 that fine-tunes the expression of several transmembrane sterol transporters involved in high-density lipoprotein (HDL) lipidation (14-17), bile metabolism (18), and hepatic vesicular secretion (19). Since SREBP2 transactivates its own promoter, miR-33 expression is increased following treatment with statins in cells and in vivo. Jeon et al. reported that SREBP2 also induces the expression of the miR-183/96/182 cluster and that miR-96 and -182 , in turn, target FBXW7 and INSIG2 thus providing feed-back regulation for SREBP2 expression and maturation (20). These studies relied on bioinformatic predictions of target genes and exogenous overexpression/inhibition of the microRNA to infer function. However, whether statins actually promote changes in the recruitment of specific mRNAs to the RISC in vivo has never been tested. 


\section{EXPERIMENTAL PROCEDURES}

Animal studies. C57BL/6 mice were obtained from NCl, housed in Optimice racks (Animal Care Systems), maintained on a 12h/12h light/dark cycle with unlimited access to food (PicoLab 5353) and water, and bred in the SPF animal facility at Saint Louis University. All mice used for in vivo experiments were male at 10-12 weeks of age. Animals used for isolation of primary hepatocytes were male or female. Where indicated, mice were gavaged $100 \mathrm{~L}$ saline or $10 \mathrm{mg} / \mathrm{kg}$ atorvastatin (Cayman Chemicals) once a day for ten days, and on the day of sacrifice once at 8 am and fasted for $6 \mathrm{~h}$ prior to tissue collection. For overexpression experiments, animals were infused via tail vein with empty or miR183/96/182 adenoviral vectors (2x109 pfu); pyruvate challenges and tissue collection were done 10- and 12-days post-infusion, respectively. For antisense experiments, animals were gavaged daily with saline or $10 \mathrm{mg} / \mathrm{Kg}$ atorvastatin as above for 6 weeks, then kept on drug while injected i.p. on days 1, 2, 3, 9, and 12 with 100 L saline, scrambled, anti-miR-96, anti-miR-182, or anti-miR-183 oligonucleotides (5 $\mathrm{mg} / \mathrm{kg}$ ) or combined anti-miR-96, -182 , and -183 oligonucleotides (1.7 mg/Kg each). Pyruvate challenges were done on day 15 at 5 am after removing food at $6 \mathrm{pm}$ the day before. Mice in the antisense experiments were not fasted before collection of blood and livers. For pyruvate challenges, baseline blood glucose was taken from tail snipping using a glucometer (Bayer Contour), then mice were injected i.p. with $2 \mathrm{~g} / \mathrm{kg}$ pyruvate, as described (21), and sequential glucose measurements were taken 15,30 , and 60 min after pyruvate injection. All animals received humane care according to the criteria outlined in the "Guide for the Care and Use of Laboratory Animals" (NIH publication 86-23 revised 1985), and all studies were approved by the IACUC at Saint Louis University.

MicroRNA vectors. Precursor miRNAs for miR-96, -182 , and -183 containing 140 bp upstream and 140 bp downstream of the mature miRNA were amplified from mouse genomic DNA using Platinum Pfx polymerase (Invitrogen), and cloned into pCMV-miR (Origene). To generate replication-deficient adenovirus, a 4,100 bp containing the whole miR cluster (from $140 \mathrm{bp}$ upstream of miR-183 to $140 \mathrm{bp}$ downstream of miR182) was amplified from mouse genomic DNA as above and cloned into pAdTrack- 
$\mathrm{CMV}$; the vector was then recombined with pAdEasy1, and transfected into Hek293Ad cells to generate high titers of replication-deficient adenovirus, as described (22).

Anti-miR oligonucleotides. 15-mer locked nucleic acid anti-ctrl (5'- TCCTAGAAAGAGTAGA), anti-miR96 (5'- ATGTGCTAGTGCCAA), anti-miR-182 (5'-TTCTACCATTGCCAA), anti-miR-183 (5'-

TTCTACCAGTGCCAT) oligonucleotides were a kind gift from Miragen Therapeutics Inc. Antimirs were resuspended in sterile saline and stored at $-20^{\circ} \mathrm{C}$ until used.

Primary hepatocytes. Cells were isolated from 8-10-week-old, male C57BL/6 mice fed chow, using Perfusion and Digest buffers (Invitrogen). Cells were resuspended in William's E Medium (Invitrogen) supplemented with Plating Supplements (Invitrogen), plated in 12- or 6-well BioCoat Collagen I plates (BD), and incubated at $37^{\circ} \mathrm{C}$ and $5 \% \mathrm{CO}_{2}$ for $6 \mathrm{~h}$. Then, the media was switched to William's $\mathrm{E}$ supplemented with Maintenance Supplements (Invitrogen). Where indicated, cells were cultured in media supplemented with $1 \mu \mathrm{mol} / \mathrm{L}$ atorvastatin or pravastatin, $5 \mu \mathrm{mol} / \mathrm{L}$ simvastatin (Cayman), or $10 \mathrm{nmol} / \mathrm{L}$ siRNA-control or siRNA-Tcf712 (Dharmacon D-001810 and L-058892, respectively). Glucose production assays were performed as described (23). Briefly, cells were incubated $6 \mathrm{~h}$ in $1 \mathrm{~mL}$ glucose production buffer (glucose-free DMEM without phenol red supplemented with $20 \mathrm{mmol} / \mathrm{L}$ sodium lactate and 2 $\mathrm{mmol} / \mathrm{L}$ sodium pyruvate. Supernatants were collected and used to measure glucose with a colorimetric kit (Wako), and data were normalized to intracellular protein.

Luciferase reporter assays. HEK293 cells were transiently transfected with luciferase reporters and miR expression plasmids in triplicate in 24-well plates using the calcium phosphate method. Cell extracts were prepared $48 \mathrm{~h}$ later, and luciferase activity measured using the Luciferase Assay System (Promega). Data were normalized to $\beta$-galactosidase activity to correct for small changes in transfection efficiency. 
RNA analysis. RNA was extracted from mouse livers and cells using Trizol (Invitrogen). RISCassociated RNA was immunoprecipitated from liver homogenates using an anti-AGO2 antibody (Wako, clone 2D4) and extracted as described (24). Bar-coded libraries for small and polyA-enriched large RNAs, and for RISC-associated RNAs were prepared for livers from saline $(n=8)$ and atorvastatin $(n=8)$ treated mice using Illumina kits, and transcriptional profiling by deep sequencing were analyzed as described $(24,25)$. For coding transcriptome, data from Saline-4 and Atorvastatin-2 libraries were discarded due to poor sequencing. For miRNome, all 16 libraries produced good quality data. For RISCome, libraries for Saline-1, -3, -4, -5, and 6, and for Atorvastatin-3, -4, -5, and -7 produced quality data. Annotated sequencing data are available in Supplemental Files 1-3. Predicted miRNA-mRNAs pairs/networks were evaluated with miRTarVis, as described (26). For all other experiments, cDNAs were generated from $1 \mu \mathrm{g}$ of DNase1-treated RNA using Superscript III (Invitrogen) and random hexamers. Quantitative real-time PCR was done with Power SybrGreen reagent (Applied Biosystems), using a LightCycler-480 (Roche). Primer sets are available upon request. Values were calculated using the comparative $\Delta \Delta \mathrm{C}_{\mathrm{t}}$ method and normalized to ribosomal Rplpo. Targeted miRNA expression was analyzed using miRCURY LNA reagents and assays (Exiqon) and normalized to U6.

Protein analysis. Protein were extracted from cells or livers using HEPES buffer $(20 \mathrm{mmol} / \mathrm{L} \mathrm{HEPES} \mathrm{pH}$ 7.4, $150 \mathrm{mmol} / \mathrm{L} \mathrm{NaCl}, 0.2 \mathrm{mmol} / \mathrm{L}$ EDTA, $2 \mathrm{mmol} / \mathrm{L} \mathrm{MgCl} 2,1 \%$ Triton X-100, $10 \%$ glycerol) containing protease inhibitor cocktail (Pierce). Forty micrograms of protein were resolved in 4-12\% polyacrylamide gels, transferred to PVDF membranes, and probed with antibodies for TCF7L2 (1:1,000 dilution, Cell Signaling C48H11), PCK1 (1:1,000 dilution; Abcam ab70358), G6PC (1:1,000 dilution; Novus NBP180533) and $\beta$-ACTIN (1:5,000 dilution; SCBT sc-130656) in TBS-Tween20 containing 4\% non-fat dry milk. Immune complexes were detected with horseradish peroxidase-conjugated anti-rabbit secondary antibodies (1:5,000 dilution; Bio-Rad 1706515). 


\section{RESULTS}

In an effort to understand the functional changes in the hepatic transcriptome that follow treatment with statins, we dosed C57BL/6 mice with saline or atorvastatin, and use RNA-seq to define total mRNAs (coding transcriptome), mRNAs associated with the RISC (RISCome), and miRNAs (miRNome) (Fig. 1a). The complete annotated sequencing data are available in Supplementary Files 13. Fig. $1 \mathrm{~b}$ shows that $\sim 10 \%$ of the coding transcriptome changed in response to the drug. As expected, at least 15 canonical targets of SREBP2 such as $H m g c r$, squalene synthase ( $F d f t 1)$, lanosterol synthase (Lss), 7- and 24-dehydrocholesterol reductases (Dhcr7, Dhcr24), proprotein convertase subtilisin/kexin type 9 (Pcsk9), and LDL-receptor (Ldlr) were induced in the livers of atorvastatin-treated mice, compared to saline (Supplementary File 1). Data in Figs. 1c and 1d, and in Supplementary Files 2 and 3 show that 62 miRNAs and 1,504 RISC-associated mRNAs were differentially regulated by the statin. Our ultimate goal, however, was to identify transcripts that were both reciprocally regulated in total transcriptome vs. RISCome, and predicted as targets of miRNAs differentially regulated in the miRNome. Analysis of the three sets of data revealed several miR/mRNA pairs that fit those criteria (Fig. 1e and Supplemental Data Fig. 1). In the rest of this report, we focus on the miR-183/96/182 polycistronic cluster, because all 3 miRNAs were upregulated in the livers of mice treated with atorvastatin, compared to saline (Fig. 1c), and 9 predicted targets for one or more miRNAs in that cluster were both RISC-enriched and transcriptome-depleted in the same livers (Fig. 1e). These targets are Bicd2 (bicaudal D homolog 2), Mkl2 (myocardin-like 2), Nox4 (NADPH oxidase 4), Pik3r1 (phosphoinositide-3-kinase regulatory subunit 1), Pxmp4 (peroxisomal membrane protein 4), Sfxn5 (sideroflexin 5), Slc22a23 (solute carrier family 22 member 23), Tcf7l2 (transcription factor 7 like 2), and Tmem189 (transmembrane protein 189). Data in Figs. $2 a$ and $2 b$ show the relative abundance of miR-183, -96 , and -182 in the livers of saline-treated mice, and the fold-induction following treatment with saline. We also highlight miR-33 in the same panels, as we and others have previously described this miRNA as regulated by statins. Data in Fig. 2c show the transcriptome and RISCome scores for the nine potential targets defined above. Importantly, both the induction of the miR cluster and the repression of the nine putative targets in response to statins were 
validated by RT-qPCR in a second cohort of mice (data not shown) and in mouse primary hepatocytes (Fig. 2d). Collectively, data in Figs. 1 and 2 demonstrate that miR-183/96/182 is an in vivo hepatic sensor of exposure to statins, and that these miRNAs actively recruit specific mRNA targets to the RISC in response to statins.

In agreement with a report by Jeon et al. (20), we mapped two SREBP-responsive elements (SRE) upstream of the transcription start for the miR-183/96/182 cluster (Supplemental Data Fig. 2a). The proximal element is evolutionarily conserved between mice and humans, while the distal one is divergent (Supplemental Data Fig. 2b). Nevertheless, both sequences in the human and mouse promoters confer responsiveness to SREBP2 (Supplemental Data Fig. 2c, d).

Of the nine transcripts that originally fit our criteria (Figs. 1e and 2c), we decided to focus on Tcf7/2, as it has been identified as a key transcriptional regulator of hepatic gluconeogenesis, and polymorphisms in this locus have been associated with diabetes in several GWAS (27-31). We hypothesized that statins stimulate hyperglycemia, at least in some patients, by increasing hepatic glucose output via miR-183/96/182-TCF7L2. The miRNA target prediction algorithm Targetscan (32) identifies conserved sequences in the 3' UTR of TCF7L2 that are partially complementary to miR-183, 96, -or 182 (Fig. 3a). These sequences, or the entire 3' UTR of mouse or human TCF7L2, were cloned downstream of a luciferase reporter and transiently transfected into HEK293 cells in the presence or absence of a plasmid encoding each miR. Data from these experiments in Fig. 3b show that each isolated conserved sequence (but not sequence 1 in human TCF7L2, which is not conserved in mouse), as well as the whole 3' UTR, were able to confer responsiveness to miR-183, -96 , and -182. Additionally, point mutations in each predicted sequence to prevent interaction with the seed sequence of each miRNA abrogated the response (Supplemental Data Fig. 3). Importantly, data in Fig. 3c using anti-miR oligonucleotides demonstrate that in mouse primary hepatocytes the miR-183/96/182 cluster mediates the repressive effect of atorvastatin on Tcf7/2. Together, data in Fig. 3 identify conserved sequences in TCF7L2 as functional statin and miR-183/96/182 responsive elements. 
We next tested the ability of statins, the miR cluster, and TCF7L2 to modulate glucose production

in mouse primary hepatocytes. Data in Fig. 4 show that gluconeogenesis was increased in cells incubated with either atorvastatin, simvastatin, or pravastatin, compared to vehicle (Fig. 4a); in cells overexpressing miR-183/96/182 (via adenoviral transduction), compared to empty vector (Fig. 4b); and in cells where Tcf7l2 was silenced using siRNA, compared to control oligonucleotides (Fig. 4c). Consistent with these data from cultured hepatocytes, the amounts of the key gluconeogenic enzymes phosphoenolpyruvate kinase 1 (PCK1) and glucose-6-phosphatase, catalytic subunit (G6PC) increased in the livers dosed with atorvastatin, compared to saline, and were inversely correlated to those of TCF7L2 (Fig. 4d). Collectively, these data demonstrate that statins, the miR cluster, and TCF7L2 are critical regulators of the gluconeogenic pathway in cultured hepatocytes.

To test the functional consequences of miR-183/96/182 overexpression on hepatic glucose production in vivo, we transduced mice with empty adenovirus or adenovirus encoding the miR cluster. After 10 days, animals were fasted overnight and then injected i.p. with pyruvate early in the morning. Hepatic gluconeogenic capacity was estimated from the conversion of pyruvate to glucose and its release into circulation, which was monitored with a standard glucometer. Data in Fig. 5a and Supplemental Data Fig. 4 show that, in two independent experiments, mice overexpressing the miR cluster were able to sustain increased glycemia in response to the pyruvate challenge, compared to control animals. These data demonstrate increased gluconeogenic capacity in the former mice. Animals were allowed to recover after the pyruvate test for $48 \mathrm{~h}$, and then some were fasted overnight, and some allowed access to food. In agreement with the pyruvate challenge results, both mRNA (Fig. 5b and Supplemental Data Fig. 4b) and protein (Fig. 5c) panels show that miR overexpression in the liver resulted in the decrease of TCF7L2 and the concomitant increase in PCK1 and G6PC. These results are consistent with the previously reported suppressive effect of TCF7L2 on the gluconeogenic pathway via negative regulation of $P C K 1$ and $G 6 P C$ expression (see discussion below). Interestingly, these changes were much more apparent in the livers of fed mice, and indeed PCK1 and G6PC proteins were as robustly expressed (if not more) in the livers of fed miR-overexpressing mice as in fasted control livers 
(Fig. 5c). This latter observation suggests that the miR-183/96/182-TCF7L2 pathway can overcome the canonical nutritional regulation of these gluconeogenic genes.

Finally, we tested whether therapeutic silencing of each miR (or of all three) could revert the atorvastatin-induced increase in hepatic gluconeogenesis. Mice were randomized for daily gavage with saline or atorvastatin during 6 weeks, and then kept on treatment while dosed with one or more anti-miR oligonucleotides on days 1, 2, 3, 9 and 12 (see Methods for details). Data in Fig. 5d show that the statin (or statin plus scrambled anti-miR), raised the expression of all three miRNAs in liver, and that the antimiRs were specific in silencing the expression of each cognate miR. Interestingly, silencing miR-96 modestly reduced the expression of miR-183, and vice-versa. This is consistent with reports in other multi-cistronic miRNAs where altering the expression of one miR affects the levels of the others in the cluster. Nonetheless, Tcf7l2 mRNA (Fig. 5d) and protein (Fig. 5e) levels were efficiently reduced in the livers of mice dosed with the statin, or statin plus scrambled anti-miR. Importantly, silencing miR-96 or miR-183, but not miR-182, negated the statin-induced reduction in Tcf7/2 both at the mRNA (Fig. $5 \mathrm{~d}$ ) and protein (Fig. 5e) level. Silencing all three miRNAs did not provide further increase in TCF7L2 abundance. Supplemental Data Fig. 5a show that the expression of G6pc in the same livers largely opposed that of Tcf7l2: both gluconeogenic transcripts were induced by the statin, and treatment with anti-miRs abrogated the statin-dependent induction. Together, these data demonstrate that the miR-183/96/182 cluster is the in vivo mediator of the statin-induced regulation of hepatic TCF7L2 and gluconeogenic enzymes. Interestingly, anti-miR182 was able to reduce the expression of G6pc (Supplemental Data Fig. 5a), despite having no effect on Tcf7/2 levels (Fig. 5c, d). No miR-182 binding sites are predicted in murine or human G6PC. Thus, the mechanism by which miR-182 modulates G6pc expression independent of Tcf7l2 remains unknown. Supplemental Data Fig. $5 a$ also shows results for the other 8 transcripts initially identified in this study: data demonstrate that the miR-183/96/182 cluster does indeed control the liver expression of Sfxn5, Tmem189, Slc22a23, Nox4, and Mkl2; however, no changes in the mRNA amounts of Pi3kr1, Pxmp4, and Bicd2 were noted. The reasons for the lack of response to atorvastatin of these latter genes are unclear, since both transcripts clearly were regulated in other in 
vivo (Fig. 1) and in culture (Fig. 2d) experiments. It is possible that the nutritional status of the mice (animals were not fasted in Fig. 5d-f and Supplemental Data Fig. 5, as opposed to those in Figs. 1 and 2) may contribute additional regulatory mechanisms, beyond SREBP2, for these genes. Nonetheless, Fig. $5 f$ shows data from the same animals two days before sacrifice, when they were challenged with pyruvate to measure in vivo gluconeogenesis. Consistent with the data in Fig. 5a from miRoverexpressing mice, hepatic glucose production was induced in mice dosed with atorvastatin (or atorvastatin plus scrambled anti-miR), compared to animals receiving saline; importantly, treatment with anti-miRs abrogated the effect of the statin and returned glycemic kinetics to control levels. These latter results upon pyruvate challenge were reproducible in a second cohort of mice treated as above

\section{DISCUSSION}

The cardiovascular therapeutic benefits of statins are presumed to outweigh their diabetogenic effect. Yet, it is critically important to establish the molecular mechanisms behind this undesired side effect. This is especially important when considering preventive prescription to younger dyslipidemic patients who will presumably be taking statins for several decades. Clinical and in vitro studies showed that statins impair both pancreatic insulin secretion and peripheral insulin sensitivity $(4,5)$. Ours is the first report demonstrating a direct effect of statins on hepatic glucose production. We show that the SREBP2-miR183/96/182-TCF7L2 axis controls the expression of gluconeogenic enzymes, and increases glucose production both in cultured hepatocytes and in mice (Fig. 6). Additionally, we show that therapeutic silencing of the miR cluster normalizes Tcf7/2 expression and reverts statin-stimulated hepatic glucose production. TCF7L2 is a member of the Wnt signaling pathway (33), and modulates the transcription of target genes by interacting not only with $\beta$-catenin (its canonical partner), but also with the key metabolic transcription factors FOXO1 (34) and HNF4 $\alpha$ (35). The exact role of TCF7L2 on glucose metabolism has 
been controversial for some time (36), and authors suggested that TCF7L2 acts as a transcriptional activator or repressor depending on its partner. In addition, at least 13 different transcripts and 2 different sized proteins are expressed from TCF7L2, and it is unclear whether these isoforms differ in their transactivating/repressing properties. Four different $T c f 72^{-1-}$ models have been generated, all with perinatal lethality(34, 37-39). Oh et al. showed that $T c f 72^{+/-}$mice display decreased glucose tolerance and insulin sensitivity, and exhibited increased hepatic expression of gluconeogenic genes (34). In contrast, Savic et al.(38) and Boj et al.(39) reported improved glycemic control using two other heterozygote mouse models and a BAC transgenic mouse, but did not report on hepatic glucose production. The reasons for the discrepancies among these different genetic models remain unknown. More recently, a liver-specific transgenic mouse expressing a dominant negative form of TCF7L2 showed progressive impairment in response to pyruvate challenge in the absence of hepatic insulin intolerance (40). Collectively, these studies show that TCF7L2 activity modulates hepatic and peripheral glucose metabolism, and wholebody glycemic control. In regards to gluconeogenesis, the literature strongly suggests that TCF7L2 reduces hepatic gluconeogenesis likely by decreasing the transcriptional activity of positive regulators of PCK1 and G6PC (34-36, 41, 42).

Interestingly, TCF7L2 transcription is stimulated postprandially by insulin (41). One key observation in our studies is that the induction miR-183/96/182 overrides the canonical nutritional control of the gluconeogenic program (on during fasting, off postprandially). As a consequence, we demonstrate that both the mRNA and protein amounts of both PCK1 and G6PC are similar in fed miR-overexpressing livers than in fasted control livers. This has particularly worrisome implications when considering patients taking statins for extended periods of time, whom presumably will have persistently elevated expression of the miR cluster: our results suggest that these patients may have sustained activation of the gluconeogenic pathway. In an otherwise healthy individual, this accelerated glucose delivery from liver into circulation should trigger homeostatic insulin-dependent clearance in peripheral tissues to maintain euglycemia. However, it is tempting to speculate that the sustained unregulated hepatic glucose production in response to statins may over time elevate blood $\mathrm{HbA} 1 \mathrm{c}$ and fasting glucose, and ultimately 
contribute to new-onset diabetes in "vulnerable" patients. Meta-analyses of clinical trials and populationbased studies revealed statin therapy increases the risk of new-onset diabetes up to $43 \%$. However, why only some, but not all, patients taking statins develop diabetes remains a mystery. Additional research is necessary to determine whether these "vulnerable" patients, who are prone to develop statin-induced diabetes, have polymorphisms in the miR-183/96/182 or TCF7L2 loci that make them more sensitive (i.e., hyper-responsive) to statins.

\section{REFERENCES}

1. Steinberg D. Thematic review series: the pathogenesis of atherosclerosis. An interpretive history of the cholesterol controversy, part V: the discovery of the statins and the end of the controversy. J Lipid Res 2006;47:1339-1351.

2. Brown MS, Goldstein JL. A proteolytic pathway that controls the cholesterol content of membranes, cells, and blood. Proceedings of the National Academy of Sciences of the United States of America 1999;96:11041-11048.

3. Liao JK, Laufs U. Pleiotropic effects of statins. Annual review of pharmacology and toxicology 2005;45:89-118.

4. Betteridge DJ, Carmena R. The diabetogenic action of statins - mechanisms and clinical implications. Nat Rev Endocrinol 2016;12:99-110.

5. Laakso M, Kuusisto J. Diabetes Secondary to Treatment with Statins. Curr Diab Rep 2017;17:10.

6. Xia F, Xie L, Mihic A, Gao X, Chen Y, Gaisano HY, Tsushima RG. Inhibition of cholesterol biosynthesis impairs insulin secretion and voltage-gated calcium channel function in pancreatic betacells. Endocrinology 2008;149:5136-5145.

7. Chamberlain LH. Inhibition of isoprenoid biosynthesis causes insulin resistance in 3T3-L1 adipocytes. FEBS Lett 2001;507:357-361.

8. Nakata M, Nagasaka S, Kusaka I, Matsuoka H, Ishibashi S, Yada T. Effects of statins on the adipocyte maturation and expression of glucose transporter 4 (SLC2A4): implications in glycaemic control. Diabetologia 2006;49:1881-1892.

9. Kwak HB, Thalacker-Mercer A, Anderson EJ, Lin CT, Kane DA, Lee NS, Cortright RN, et al. Simvastatin impairs ADP-stimulated respiration and increases mitochondrial oxidative stress in primary human skeletal myotubes. Free Radic Biol Med 2012;52:198-207.

10. Galtier F, Mura T, Raynaud de Mauverger E, Chevassus H, Farret A, Gagnol JP, Costa F, et al. Effect of a high dose of simvastatin on muscle mitochondrial metabolism and calcium signaling in healthy volunteers. Toxicol Appl Pharmacol 2012;263:281-286. 
11. Bartel DP. MicroRNAs: target recognition and regulatory functions. Cell 2009;136:215-233.

12. van Rooij E, Kauppinen S. Development of microRNA therapeutics is coming of age. EMBO Mol Med 2014;6:851-864.

13. Baldan A, Fernandez-Hernando $C$. Truths and controversies concerning the role of miRNAs in atherosclerosis and lipid metabolism. Curr Opin Lipidol 2016;27:623-629.

14. Marquart TJ, Allen RM, Ory DS, Baldan A. miR-33 links SREBP-2 induction to repression of sterol transporters. Proc Natl Acad Sci U S A 2010;107:12228-12232.

15. Rayner KJ, Suarez Y, Davalos A, Parathath S, Fitzgerald ML, Tamehiro N, Fisher EA, et al. miR-33 Contributes to the Regulation of Cholesterol Homeostasis. Science 2010;238:1570-1573.

16. Horie T, Ono K, Horiguchi M, Nishi H, Nakamura T, Nagao K, Kinoshita M, et al. MicroRNA-33 encoded by an intron of sterol regulatory element-binding protein 2 (Srebp2) regulates HDL in vivo. Proc Natl Acad Sci U S A 2010;107:17321-17326.

17. Najafi-Shoushtari SH, Kristo F, Li Y, Shioda T, Cohen DE, Gerszten RE, Naar AM. MicroRNA-33 and the SREBP Host Genes Cooperate to Control Cholesterol Homeostasis. Science 2010;238:15661569.

18. Allen RM, Marquart TJ, Albert CJ, Suchy FJ, Wang DQ, Ananthanarayanan M, Ford DA, et al. miR33 controls the expression of biliary transporters, and mediates statin- and diet-induced hepatotoxicity. EMBO molecular medicine 2012;4:882-895.

19. Allen RM, Marquart TJ, Jesse JJ, Baldan A. Control of very low-density lipoprotein secretion by Nethylmaleimide-sensitive factor and miR-33. Circ Res 2014;115:10-22.

20. Jeon TI, Esquejo RM, Roqueta-Rivera M, Phelan PE, Moon YA, Govindarajan SS, Esau CC, et al. An SREBP-responsive microRNA operon contributes to a regulatory loop for intracellular lipid homeostasis. Cell Metab 2013;18:51-61.

21. Miyake K, Ogawa W, Matsumoto M, Nakamura T, Sakaue H, Kasuga M. Hyperinsulinemia, glucose intolerance, and dyslipidemia induced by acute inhibition of phosphoinositide 3-kinase signaling in the liver. J Clin Invest 2002;110:1483-1491.

22. Luo J, Deng ZL, Luo X, Tang N, Song WX, Chen J, Sharff KA, et al. A protocol for rapid generation of recombinant adenoviruses using the AdEasy system. Nat Protoc 2007;2:1236-1247.

23. Yoon JC, Puigserver P, Chen G, Donovan J, Wu Z, Rhee J, Adelmant G, et al. Control of hepatic gluconeogenesis through the transcriptional coactivator PGC-1. Nature 2001;413:131-138.

24. Matkovich SJ, Van Booven DJ, Eschenbacher WH, Dorn GW, 2nd. RISC RNA sequencing for context-specific identification of in vivo microRNA targets. Circ Res 2011;108:18-26.

25. Matkovich SJ, Zhang Y, Van Booven DJ, Dorn GW, 2nd. Deep mRNA sequencing for in vivo functional analysis of cardiac transcriptional regulators: application to Galphaq. Circ Res 2010;106:1459-1467.

26. Jung D, Kim B, Freishtat RJ, Giri M, Hoffman E, Seo J. miRTarVis: an interactive visual analysis tool for microRNA-mRNA expression profile data. BMC Proc 2015;9:S2. 
27. Grant SF, Thorleifsson G, Reynisdottir I, Benediktsson R, Manolescu A, Sainz J, Helgason A, et al. Variant of transcription factor 7-like 2 (TCF7L2) gene confers risk of type 2 diabetes. Nature genetics 2006;38:320-323.

28. Sladek R, Rocheleau G, Rung J, Dina C, Shen L, Serre D, Boutin P, et al. A genome-wide association study identifies novel risk loci for type 2 diabetes. Nature 2007;445:881-885.

29. Cauchi S, El Achhab Y, Choquet H, Dina C, Krempler F, Weitgasser R, Nejjari C, et al. TCF7L2 is reproducibly associated with type 2 diabetes in various ethnic groups: a global meta-analysis. Journal of molecular medicine 2007;85:777-782.

30. Zhang C, Qi L, Hunter DJ, Meigs JB, Manson JE, van Dam RM, Hu FB. Variant of transcription factor 7-like 2 (TCF7L2) gene and the risk of type 2 diabetes in large cohorts of U.S. women and men. Diabetes 2006;55:2645-2648.

31. Damcott CM, Pollin TI, Reinhart LJ, Ott SH, Shen H, Silver KD, Mitchell BD, et al. Polymorphisms in the transcription factor 7-like 2 (TCF7L2) gene are associated with type 2 diabetes in the Amish: replication and evidence for a role in both insulin secretion and insulin resistance. Diabetes 2006;55:2654-2659.

32. Agarwal V, Bell GW, Nam JW, Bartel DP. Predicting effective microRNA target sites in mammalian mRNAs. Elife 2015;4.

33. Wiese KE, Nusse R, van Amerongen R. Wnt signalling: conquering complexity. Development $2018 ; 145$.

34. Oh KJ, Park J, Kim SS, Oh H, Choi CS, Koo SH. TCF7L2 modulates glucose homeostasis by regulating $\mathrm{CREB}$ - and FoxO1-dependent transcriptional pathway in the liver. PLoS genetics 2012;8:e1002986.

35. Neve B, Le Bacquer O, Caron S, Huyvaert M, Leloire A, Poulain-Godefroy O, Lecoeur C, et al. Alternative human liver transcripts of TCF7L2 bind to the gluconeogenesis regulator HNF4alpha at the protein level. Diabetologia 2014;57:785-796.

36. Jin T. Current Understanding on Role of the Wnt Signaling Pathway Effector TCF7L2 in Glucose Homeostasis. Endocr Rev 2016;37:254-277.

37. Korinek V, Barker N, Moerer P, van Donselaar E, Huls G, Peters PJ, Clevers H. Depletion of epithelial stem-cell compartments in the small intestine of mice lacking Tcf-4. Nature genetics 1998;19:379-383.

38. Savic D, Ye H, Aneas I, Park SY, Bell GI, Nobrega MA. Alterations in TCF7L2 expression define its role as a key regulator of glucose metabolism. Genome research 2011;21:1417-1425.

39. Boj SF, van Es JH, Huch M, Li VS, Jose A, Hatzis P, Mokry M, et al. Diabetes Risk Gene and Wnt Effector Tcf7I2/TCF4 Controls Hepatic Response to Perinatal and Adult Metabolic Demand. Cell 2012;151:1595-1607.

40. Ip W, Shao W, Song Z, Chen Z, Wheeler MB, Jin T. Liver-specific expression of dominant-negative transcription factor 7 -like 2 causes progressive impairment in glucose homeostasis. Diabetes 2015;64:1923-1932. 
41. Ip W, Shao W, Chiang YT, Jin T. The Wnt signaling pathway effector TCF7L2 is upregulated by insulin and represses hepatic gluconeogenesis. American journal of physiology. Endocrinology and metabolism 2012;303:E1166-1176.

42. Norton L, Fourcaudot M, Abdul-Ghani MA, Winnier D, Mehta FF, Jenkinson CP, Defronzo RA. Chromatin occupancy of transcription factor 7-like 2 (TCF7L2) and its role in hepatic glucose metabolism. Diabetologia 2011;54:3132-3142.

\section{FIGURE LEGENDS}

Figure 1. Atorvastatin-dependent changes in hepatic transcriptome, miRNome, and RISCome. a, Experimental design. Mice were gavaged daily with saline or $10 \mathrm{mg} / \mathrm{Kg}$ atorvastatin. See Methods for details on RNA analysis. b, Summary of changes in total coding transcriptome in atorvastatin ( $n=7)$ vs. saline ( $n=7)$. c, Summary of changes in miRNome ( $n=8 /$ group). d, Summary of changes in RISCome ( $n=5$-saline; $\mathrm{n}=4-\mathrm{ATR}$ ). e, Node-link diagram of miRTarVis-predicted miRNA-mRNA networks changing in atorvastatin vs. saline; upregulated transcripts in red, downregulated transcripts in blue. See Supplemental Files 1-3 for complete annotated sequencing data and analysis.

Figure 2. Treatment with statins induces the recruitment of miR-183/96/182 targets to the RISC. a, Relative abundance of mature miR-183, -96 , and -182 in the hepatic miRNome of saline-treated mice. For comparison, the previously reported statin-regulated miR-33 is shown in black. $\mathbf{b}$, Relative foldinduction of mature miR-183, -96 , and -182 in response to atorvastatin. c, Nine putative miR-183/96/182 targets identified in Fig. 1e were simultaneously depleted in total transcriptome and enriched in the RISCome of mice dosed with atorvastatin. $\mathbf{d}$, Validation by qPCR of changes in hepatic miR-183/96/182 and their putative targets in a second cohort of mice $(n=5)$ dosed with saline or atorvastatin, as in Figs. 1. and 2a-c. Canonical SREBP2 targets (Hmgcr, Ldlr, Pcsk9, and miR-33 are shown as positive controls). e, Validation of changes in miR-183/96/182 and their putative targets in mouse primary hepatocytes cultured $24 \mathrm{~h}$ in media supplemented with $1 \mu \mathrm{mol} / \mathrm{L}$ atorvastatin (ator) or pravastatin (pra), or $5 \mu \mathrm{mol} / \mathrm{L}$ simvastatin (smv) ( $\mathrm{n}=3$ /group). Canonical SREBP2 targets Hmgcr and miR-33 are shown as positive 
controls. Dotted line represents normalized expression in dmso-treated cells. ${ }^{*} p<0.05,{ }^{* *} p<0.01$, compared to dmso.

Figure 3. Functional miR-183/96/182-reponsive elements in TCF7L2. a, Conserved sequences in the 3'UTR of TCF7L2 with partial complementarity to miR-183/96/182 are color-coded as elements 1, 2, and 3. Distance from the stop codon are indicated. Alignments for mouse and human sequences are shown. Solid lines represent Watson-Crick complementarity; colon marks represent divergence between mouse and human sequences. b, Normalized luciferase activity in extracts from HEK293 cells transiently transfected with a reporter fused to the potential miR-responsive elements identified above or the whole 3'UTR, and expression vectors for each miR. As negative control, we used the empty pGL3Promoter vector. Data are mean \pm SE of 3 independent experiments in quadruplicate. ${ }^{* *} p<0.01$. c, miR-183/96/182 mediate atorvastatin-induced repression of Tcf7/2. Mouse primary hepatocytes were transfected with scrambled or anti-miR oligonucleotides, and $24 \mathrm{~h}$ later switched to media supplemented with vehicle or atorvastatin for $24 \mathrm{~h}$. Data are mean \pm SE of 2 independent experiments in quadruplicate. ${ }^{*} p<0.05$, compared to dmso; ${ }^{\dagger \dagger} p<0.01$, compared to atorvastatin plus anti-scr.

Figure 4. Statins, miR-183/96/182, and TCF7L2 modulate hepatic gluconeogenesis. a, Glucose production in mouse primary hepatocytes incubated in media supplemented with vehicle, $1 \mu \mathrm{mol} / \mathrm{L}$ atorvastatin, $5 \mu \mathrm{mol} / \mathrm{L}$ simvastatin, or $1 \mu \mathrm{mol} / \mathrm{L}$ pravastatin. $\mathbf{b}$, Glucose production in mouse primary hepatocytes after transduction with empty adenovirus or adenovirus encoding the miR-183/96/182 cluster. c, Glucose production in mouse primary hepatocytes after transfection with siRNA-control or siRNA-Tcf7I2 oligonucleotides. d, Hepatic protein abundance of TCF7L2 and gluconeogenic enzymes PCK1 and G6PC in mice dosed with saline or $10 \mathrm{mg} / \mathrm{Kg} / \mathrm{d}$ atorvastatin for 6 weeks. Data in panels a-c are mean \pm SE of at least 2 independent experiments in quadruplicate. ${ }^{* *} p<0.01$ 
Figure 5. Therapeutic silencing of the miR-183/96/182 cluster abrogates statin-induced hepatic

gluconeogenesis. a, Blood glucose curves in response to pyruvate challenge (see Methods) show increased hepatic glucose output in mice transduced with adenovirus encoding the miR cluster, compared to empty adenovirus. Data are mean \pm SE; $n=10 /$ group; ${ }^{*} p<0.01$. $\mathbf{b}$, Hepatic protein levels for TCF7L2, PCK1, and G6PC two days after the pyruvate challenge, in mice allowed access to food, or fasted overnight. Data are mean $\pm \mathrm{SE} ; \mathrm{n}=5$ /group; ${ }^{*} p<0.05,{ }^{* \star} p<0.01$, compared to fed mice; ${ }^{\dagger} p<0.05$, ${ }^{t \dagger} p<0.01$, compared to empty adenovirus. c, Immunoblots of hepatic protein extracts in the same mice as (b). d, Mice were gavaged with saline or $10 \mathrm{mg} / \mathrm{Kg} / \mathrm{d}$ atorvastatin daily for 6 weeks, then treated with control or anti-miR oligonucleotides, alone or combined, for 15 days (see Methods). Atorvastatin induces the hepatic expression of the $3 \mathrm{miRs}$ and reduces that of Tcf7l2. Anti-miRs are specific in silencing the expression of their cognate miR. Silencing miR-96 or -183 abolished atorvastatin-induced repression of Tcf7l2. Data are mean \pm SE; $n=6 /$ group; ${ }^{*} p<0.05,{ }^{* *} p<0.01$, compared to saline; ${ }^{\dagger} p<0.05,{ }^{\dagger} p<0.01$ compared to atorvastatin plus anti-ctrl. e, Changes in TCF7L2 protein levels parallel those in mRNA. PCSK9 was used as positive control to monitor the efficacy of the statin. $\mathbf{f}$, Hepatic gluconeogenic capacity in response to a pyruvate challenge was tested in the 4 groups shown, confirming the functional role of the miR cluster as the mediator to statin-induced hepatic glucose production. Area under the curve represented in arbitrary units. Data are mean \pm SE; $n=6 /$ group; ${ }^{*} p<0.05,{ }^{\star \star} p<0.01$, compared to saline; ${ }^{\dagger} p<0.01$, compared to atorvastatin plus anti-ctrl.

Figure 6. The SREBP-2-miR-183/96/182-TCF7L2 modulates hepatic glucose output. During episodes of low-intracellular cholesterol, such as in response to statins, the expression of miR$183 / 96 / 182$ is induced, which in turns reduces the levels of gluconeogenic repressor TCF7L2, ultimately promoting accelerated hepatic glucose production. 


\section{ACKNOWLEDGMENTS}

477 We thank Dr. Aimee Jackson at miRagen Therapeutics Inc. for providing the anti-miR oligonucleotides

478 for in vivo experiments. We also thank Dr. Cedric Langhi at NovoNordisk for discussions. This study was

479 supported in part by NIH HL107794 and AHA GRNT20460189 grants (to Á.B.), Washington University

480 Department of Medicine start-up funds (S.J.M.), and AHA PRE7240026 fellowship (to R.M.A.).

\section{AUTHOR CONTRIBUTIONS}

484 T.J.M. and Á.B. conceived the idea, designed experiments, and wrote the manuscript. T.J.M. and R.M.A. 485 performed experiments, and collected and analyzed data. M.R.C. analyzed data. G.W.D. and S.J.M. 486 supervised library construction, sequencing, and bioinformatics analysis. 


\section{Figure 1}
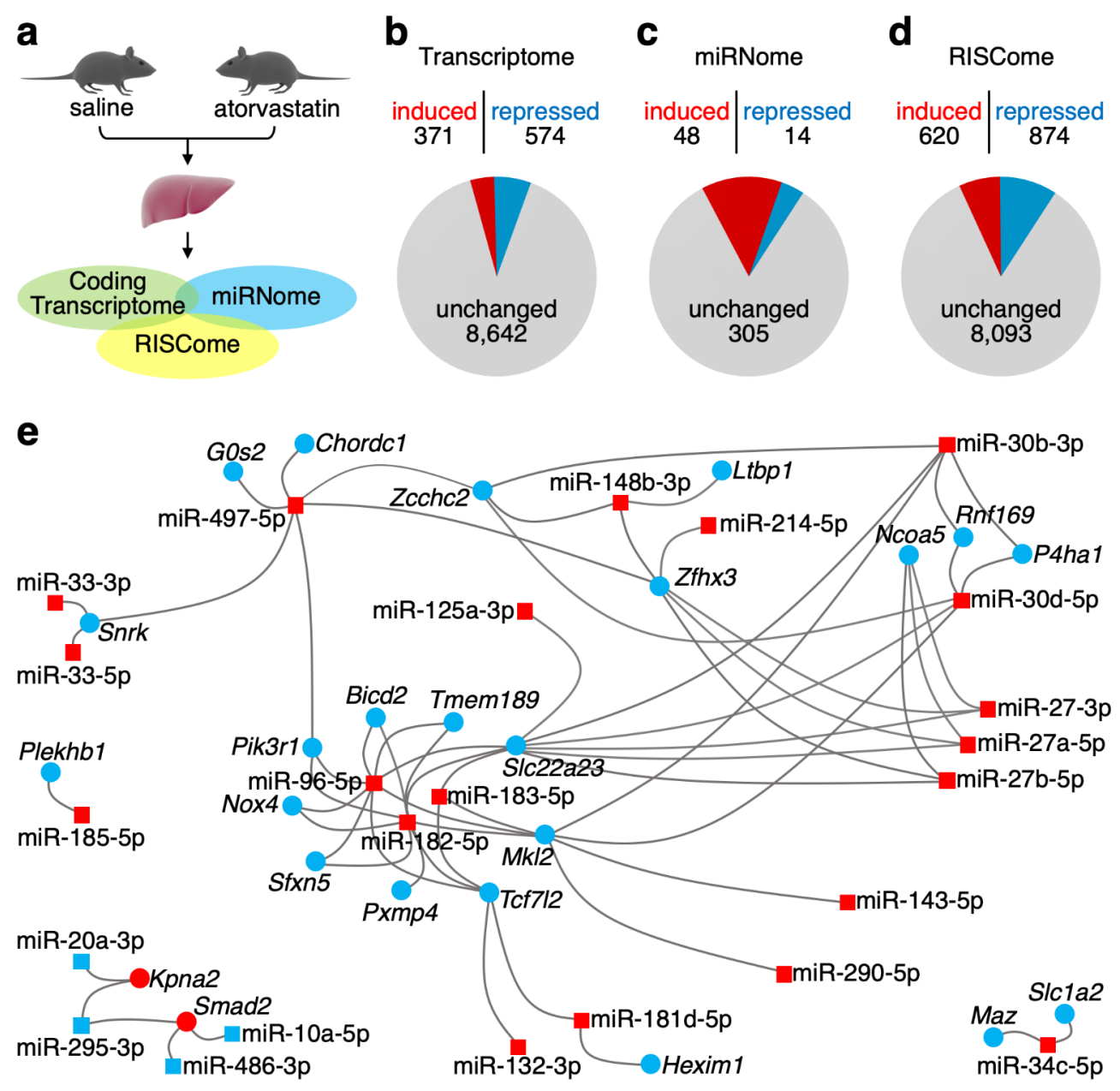


\section{Figure 2}
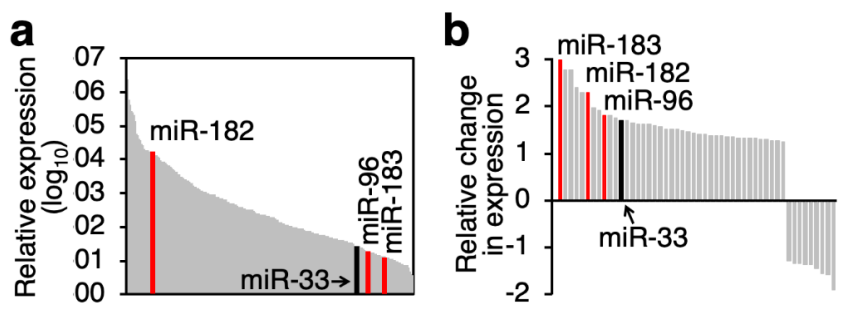

C

\begin{tabular}{|c|c|c|c|c|c|c|c|c|}
\hline \multirow{2}{*}{$\begin{array}{c}\text { Gene } \\
\text { Symbol }\end{array}$} & \multicolumn{4}{|c|}{ Transcriptome } & \multicolumn{4}{|c|}{ RISCome } \\
\hline & Saline & ATR & \begin{tabular}{|l|} 
Fold \\
\end{tabular} & $p$-value & Saline & ATR & Fold & $p$-value \\
\hline Bicd2 & 5.99 & 5.30 & 0.88 & 0.0438 & 1.61 & 2.22 & 1.38 & 0.0350 \\
\hline Mkl2 & 9.77 & 8.13 & 0.83 & 0.0444 & 7.04 & 11.03 & 1.57 & \\
\hline Nox4 & 13.47 & 10.64 & 0.79 & 0.0341 & 0.80 & 1.19 & 1.50 & \\
\hline Pik3r1 & 21.51 & 15.6 & \begin{tabular}{|l|}
0.73 \\
\end{tabular} & 0.00 & 2.32 & 2.99 & 1.32 & 0.0225 \\
\hline Pxmp4 & 61.17 & 48.19 & 0.79 & 0.0003 & 3.11 & 4.55 & 1.46 & 0.0031 \\
\hline & 24.15 & & & & 3.79 & 6.38 & 1.68 & \\
\hline SIc22a23 & 39.41 & 34. & \begin{tabular}{|l|l|}
0.87 \\
\end{tabular} & 0.0478 & 2.45 & \begin{tabular}{|l|}
3.44 \\
\end{tabular} & 1.41 & 0.0207 \\
\hline Tcf7l2 & 40.79 & 31. & 0.78 & 0.0061 & 6.01 & 8.98 & 1.49 & 0.0420 \\
\hline mem189 & 22.59 & & \begin{tabular}{|l|l|} 
& 0.78 \\
\end{tabular} & 0.0112 & 2.18 & 3.51 & 1.61 & 0.02 \\
\hline
\end{tabular}

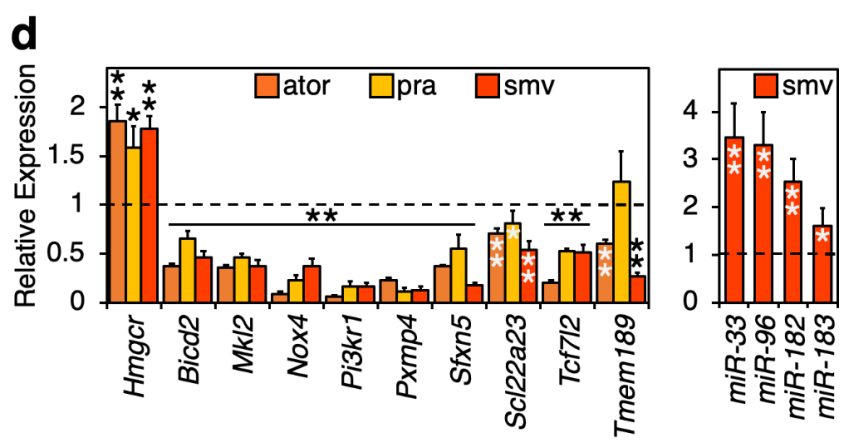




\section{Figure 3}

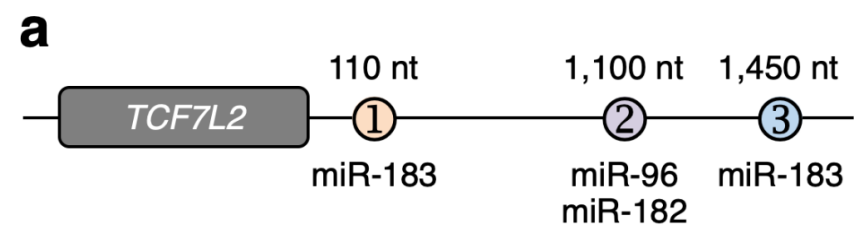

miR-183 3'-UCACUUAAGAUGGUCACGGUAU-5'
hsa UUUGUACUCUCUUAAUUUUGUGCCAU-GUGGCU
$\mathrm{mmu}$ UUUGUACUCUCUUAAUUU-GUGCCGCCGUGGCU

miR-96 3'-UCGUUUUUACACGAUCACGGUUU-5'
hsa ACCGUGUACAAAACUUU-ACGUGCCAAAAUUCU
mmu ACCGUGUGCAAAACUUUUAUGUGCCAAAAUUCU
miR-182 UCACACUCAAGAUGGUAACGGUUU-5' $(\mathrm{h})$
miR-182 GCCACACUCAAGAUGGUAACGGUUU-5
miR-183 3'-UCACUUAAGAUGGUCACGGUAU-5' : $\quad \|||||||||||$
hsa GCUGAAACCUGUUCAUUCAGUGCCAUUGUAGU $\mathrm{mmu}$ GCUGAGACCUGUUCCUUCAGUGCCAUCGUAGU

b
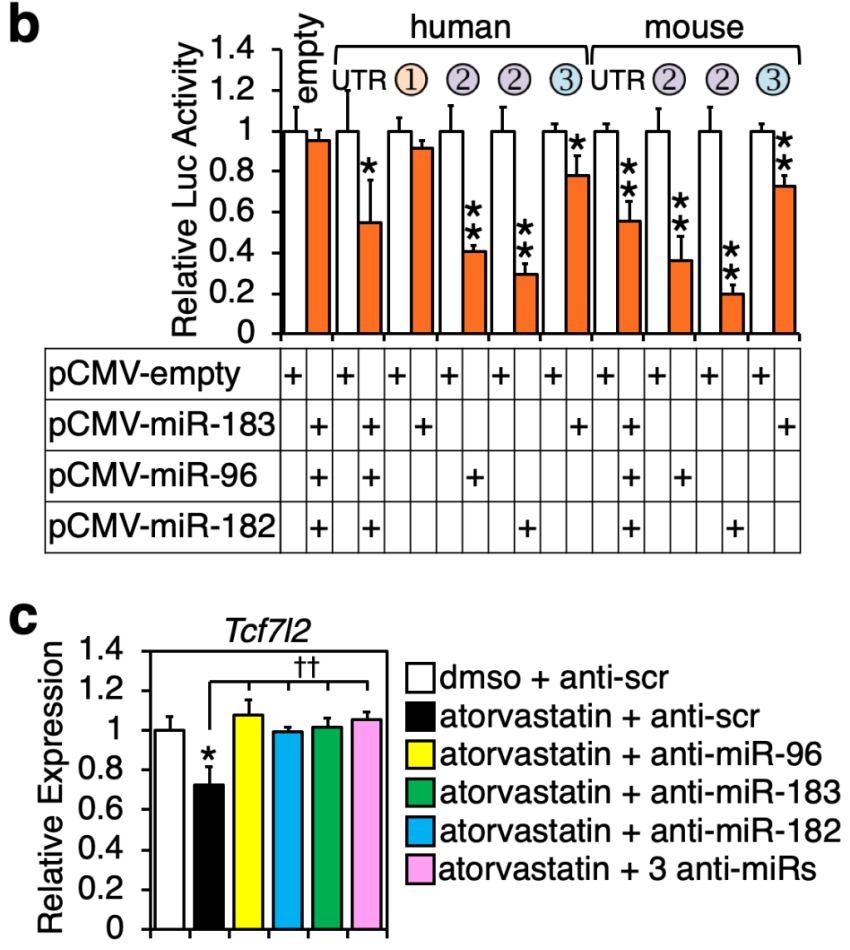


\section{Figure 4}

a

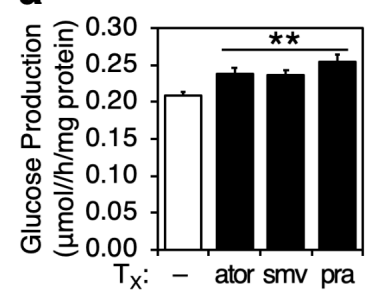

C

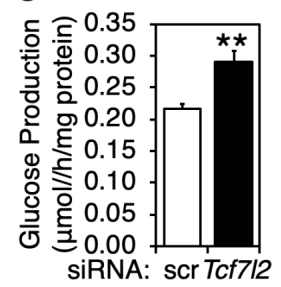

d b
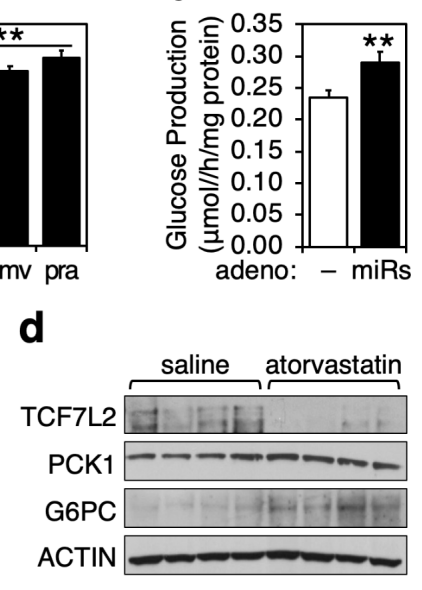


\section{Figure 5}
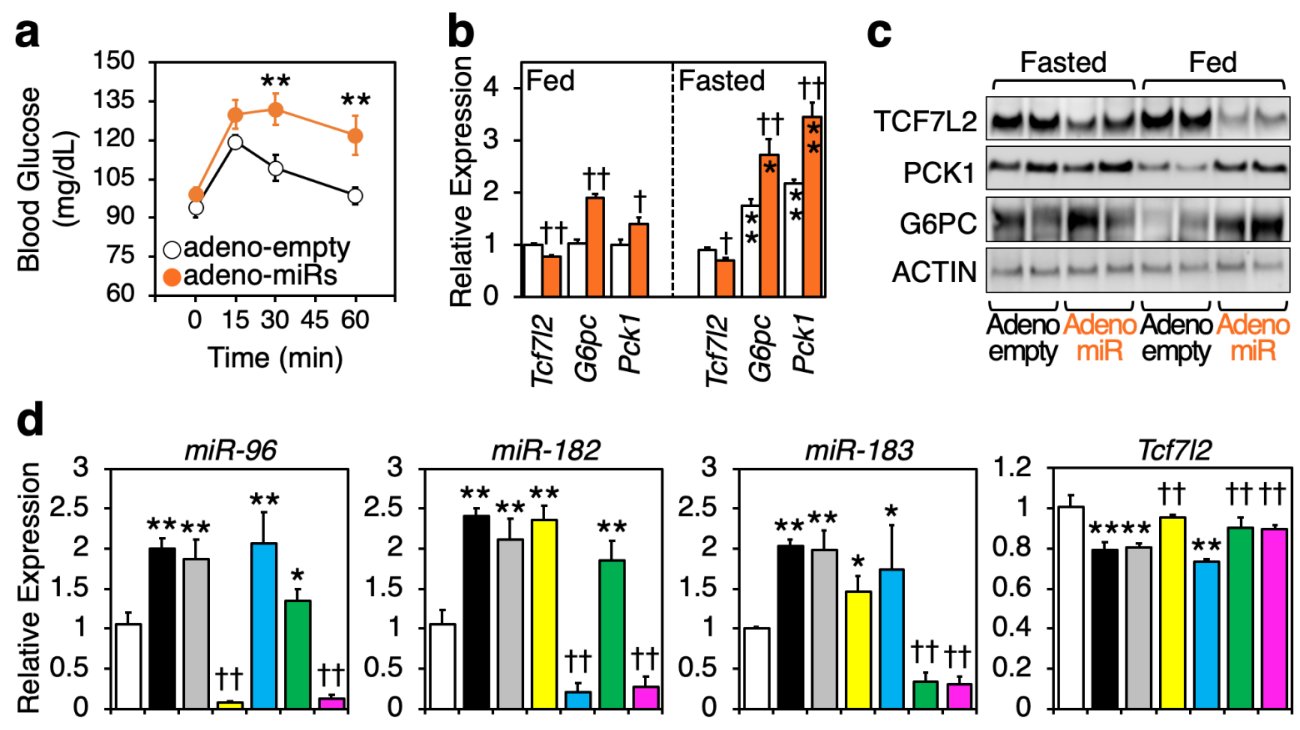

miR-182
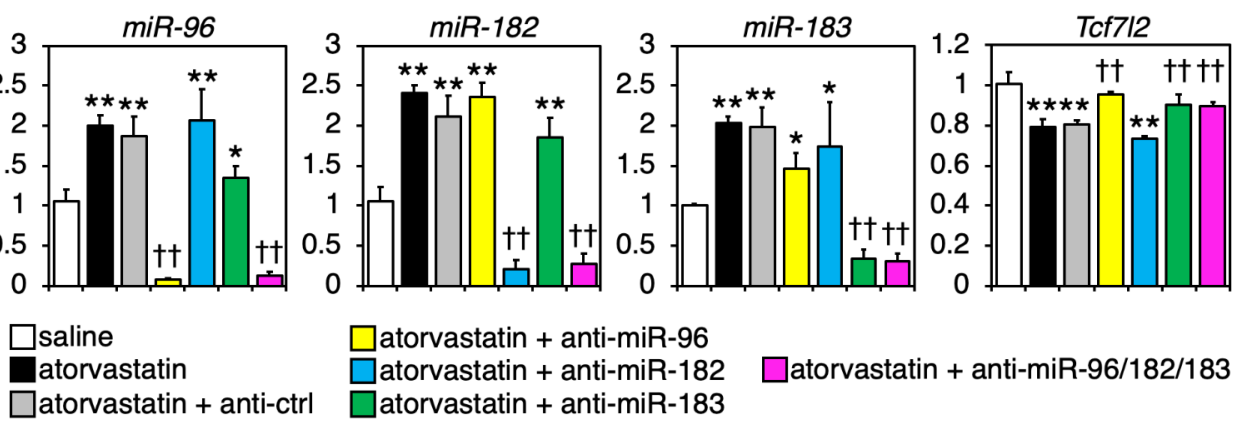

$\square$ atorvastatin + anti-miR-96

$\square$ atorvastatin + anti-miR-182

$\square$ atorvastatin + anti-miR-183
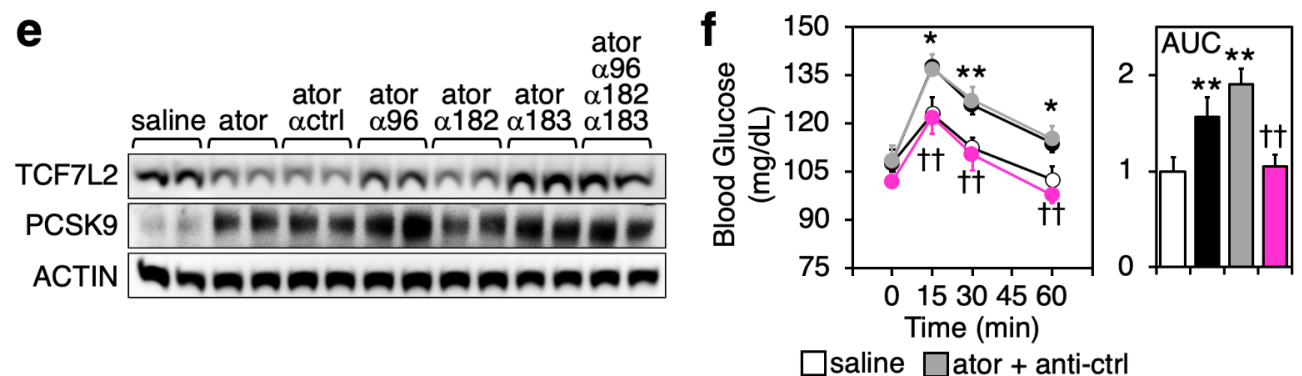

ator

$\square$ ator + anti-miR-96/182/183 


\title{
Figure 6
}

\author{
statins reduced cell \\ y \\ SREBP2 \\ $\downarrow$ \\ $\operatorname{miR}-183 / 96 / 182$ \\ $\perp$ \\ TCF7L2 \\ $\perp$ \\ PCK1/G6PC \\ $\downarrow$ \\ Hepatic Glucose Output
}

